\title{
Gamma-hadron and Related Two- Particle Azimuthal Correlations Results in PHENIX
}

\author{
Abinash Pun ${ }^{* \dagger}$ \\ Ohio University, Athens, Ohio 45701 \\ E-mail: ap564413@ohio.edu
}

\begin{abstract}
At PHENIX, direct photon-hadron and related two-particle correlations have been measured for a variety of collision systems. A photon isolation cut method is applied in $\mathrm{Au}+\mathrm{Au}$ to study jet energy loss. We present centrality-dependent isolated photon-hadron angular correlations in $\mathrm{Au}+\mathrm{Au}$ showing the evolution of enhancement and suppression behavior. In order to gain insights on jet energy loss and nuclear modifications to jets ranging from effects in small collision systems to mid-peripheral A+A systems, we also present related two-particle correlation results from small systems $\left(d+\mathrm{Au}\right.$ and $\left.{ }^{3} \mathrm{He}+\mathrm{Au}\right)$.
\end{abstract}

13th International Workshop in High pT Physics in the RHIC and LHC Era (High-pT2019) 19-22 March 2019

Knoxville, Tennessee, USA

\footnotetext{
${ }^{*}$ Speaker.

${ }^{\dagger}$ For the PHENIX Collaboration
} 


\section{Introduction}

Angular correlations between pairs of final state particles, two particle correlations, is a useful tool to study the properties of the Quark Gluon Plasma (QGP) in relativistic heavy ion collision. One of the important benefits from two particle correlations is that the contribution from jets and flow can be separated. According to a commonly used two source model, the same-event pair distribution is the sum of the distribution due to the particle pairs from hard scattering partons (jet pairs) and that due to the combinatorial background from anisotropic flow production of soft particles relative to the reaction plane. Thus,

$$
C\left(\Delta \phi_{A B}\right)=J\left(\Delta \phi_{A B}\right)+b_{0}\left[1+\sum_{n=1}^{\infty}\left\langle 2 v_{n}^{A} v_{n}^{B}\right\rangle \cos \left(n \Delta \phi_{A B}\right)\right],
$$

where $\Delta \phi$ is the azimuth difference between trigger and partner particles. The jet function, $J\left(\Delta \phi_{A B}\right)$, is the fraction of the correlation function from only jet-jet pairs. The second term in RHS of Eqn. 1.1 is from the background contribution, which is described by a Fourier series. The $b_{0}$ is the combinatorial background level which normalizes the contribution of combinatorial pairs to the correlation function.

Because of color-neutral nature of photons, direct photon-hadron correlations are an excellent choice of study for jet modification due to the QGP. We used an isolation cut [1] method to obtain the direct photons. In the isolation cut methods, a cone with a specific sized radius according to $R_{\text {cone }}=\sqrt{(\Delta \phi)^{2}+(\Delta \eta)^{2}}$ is defined around photons in each event. Then, the energy of all other particles inside the cone is measured. If the cone energy is smaller than the threshold energy, the photon is classified as isolated otherwise it is discarded. The cone threshold energy is determined by $E_{T H}=a E_{\gamma}+b$, where $E_{\gamma}$ is the photon's total energy and a and $\mathrm{b}$ are parameters varying with centrality due to the magnitude of the underlying event. This method helped to gain more statistical precision compared to the usual statistical subtraction method, and to study the more semi-peripheral and peripheral centrality bins of $\mathrm{Au}+\mathrm{Au}$ collisions. In section 2, we describe the centrality dependence of jet energy loss in $\mathrm{Au}+\mathrm{Au}$ collisions at $200 \mathrm{GeV}$ obtained from this method. Those centralities are particularly interesting to study the onset of energy loss with centrality. Absolute normalization method (ABS) [4] is used for the background subtraction in the analysis.

Also, we present an analysis of two small collision systems; $d+\mathrm{Au}$ and ${ }^{3} \mathrm{He}+\mathrm{Au}$ in section 3 . To increase statistical precision, a $\pi^{0}$ trigger is chosen and also, a new observable $\left(R_{I}\right)$ is introduced to decrease systematic uncertainties. The Zero Yield At Minimum (ZYAM) method [4] is used for the background subtraction in the analysis.

\section{Centrality Dependence of Jet Energy Loss in Au+Au using Isolation Cut Method}

The $\Delta \phi$-integrated per-trigger yield $(Y)$ for the near- and away-side regions can be used to characterize the modification of jets. The per-trigger yield nuclear modification factor, $I_{A A}$, is introduced for the comparative study of those yields between $\mathrm{Au}+\mathrm{Au}$ and $p+p$ collisions.

$$
I_{A A}=\frac{Y^{A A}}{Y^{p p}}=\frac{\int_{\Delta \phi_{1}}^{\Delta \phi_{2}}\left(\frac{1}{n_{A}} \frac{d n^{A B}}{d \Delta \phi}\right)_{A A} d \Delta \phi}{\int_{\Delta \phi_{1}}^{\Delta \phi_{2}}\left(\frac{1}{n_{A}} \frac{d n^{A B}}{d \Delta \phi}\right)_{p p} d \Delta \phi}
$$


The $I_{A A}$ might be of near- or away-side depending upon the integration range. Fig. 1 shows $\pi / 2$ integrated awayside $I_{A A}$ for different centrality regions in $\mathrm{Au}+\mathrm{Au}$ collisions. For each centrality region, the ratio has a suppression behavior in high $z_{T}$ region and enhancement in low $z_{T}$ region.

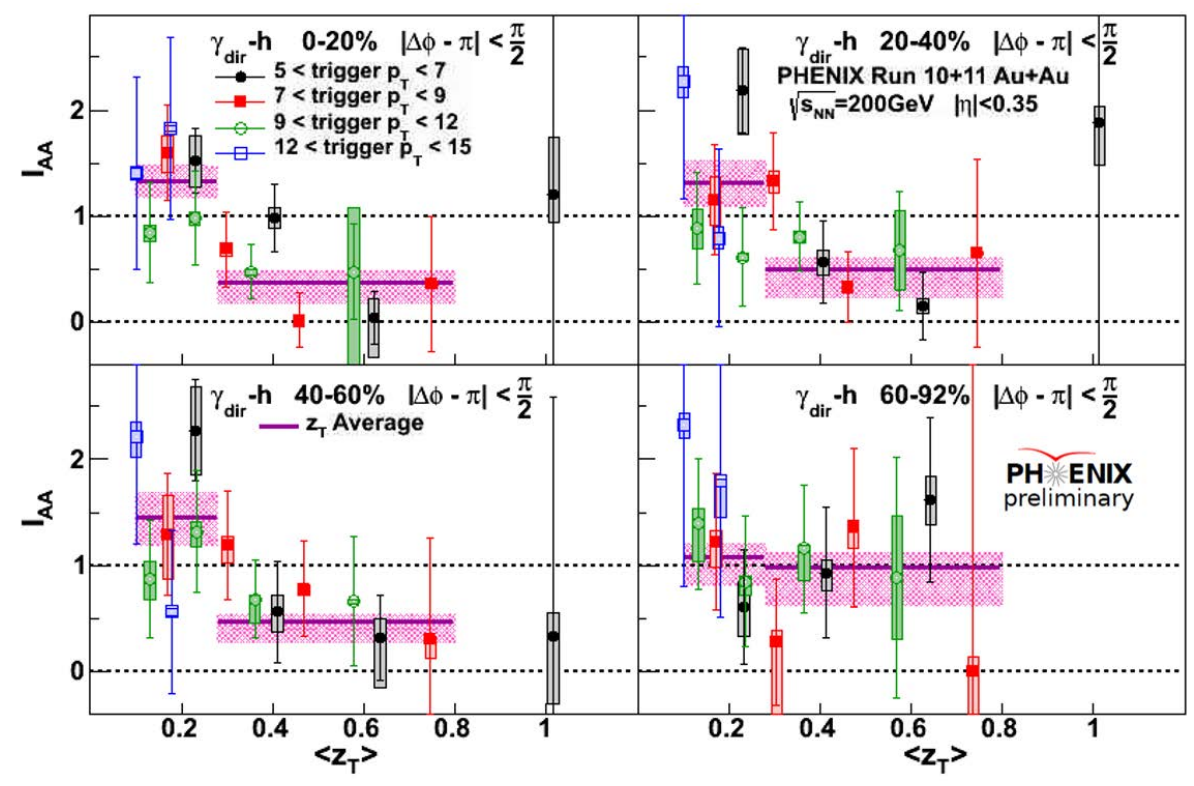

Figure 1: $I_{A A}$ for isolated $\gamma$-hadron correlation as as function of $z_{T}$ for different centralities. $z_{T}$ is ratio of transverse momentum of partner particle to that of trigger particle. The $I_{A A}$ is fitted with straight line (i.e. averaged) in two different regions. The light purple shadow around the fitting lines shows the statistical error. The figures are taken from [2].

For the study of centrality dependence of modifications in $I_{A A}$, we fit it in two $z_{T}$ regions. The centrality dependence of the modifications for different awayside integration regions are as shown in Fig. 2.
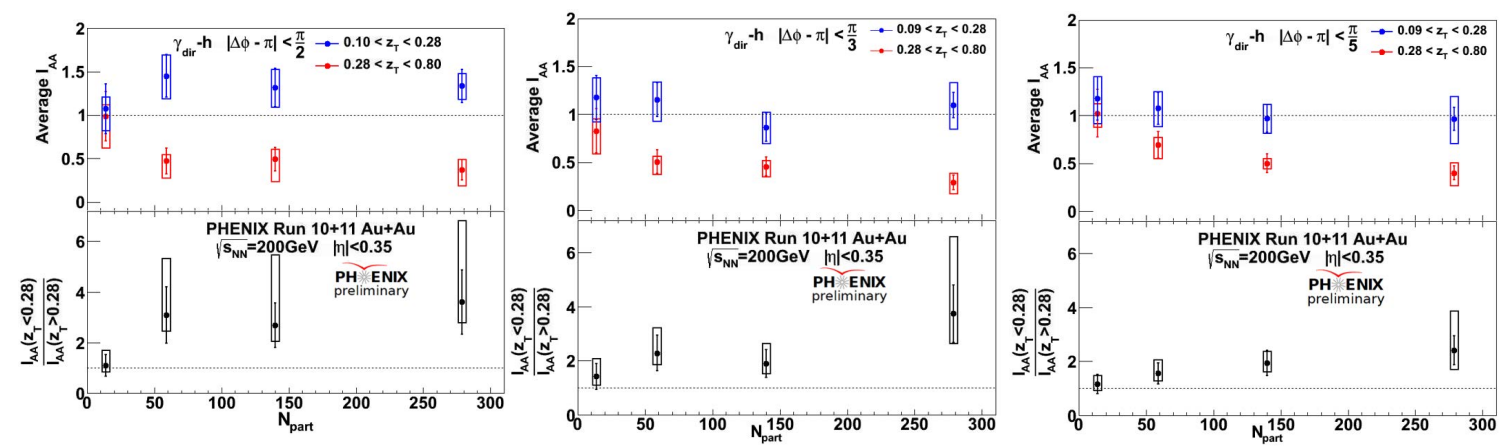

Figure 2: Upper panel: Centrality $\left(N_{\text {part }}\right)$ dependence of average $I_{A A}$ in different awayside integration regions. The red points show high $z_{T}$ behavior while blue points show low $z_{T}$ behavior. Lower panel: Relative enhancement as a function of $N_{\text {part }}$ in different awayside integration regions. The figures are taken from [2]. 
In the upper panel of Fig. 2, the blue points show high $z_{T}$ behavior while Blue shows the low $z_{T}$. There is clear high $z_{T}$ suppression and low $z_{T}$ enhancement for all integration regions. The integration region goes wider from right to left in Fig. 2. The enhancement increases as the integration range is widened. To judge the true centrality dependence of enhancement, we must account for overall reduction of jets due to suppression. For that, we take ratio of low to high $z_{T}$ points as a relative enhancement factor as it then quantifies the low $z_{T}$ modification relative to the suppression factor. The relative enhancement factor, as shown in lower panel of Fig. 2, is larger than unity for nearly all bins and reveals monotonic increase towards central events.

\section{Small Systems Collisions}

Complementary to the analysis of jet modification behavior at different centralities, especially in the most peripheral events, it would be very interesting to look for possible modification behavior in small systems. The study of the small systems can help to reveal how large a system has to be to see the energy loss.

\subsection{Double Ratio $\left(R_{I}\right)$}

The large systematic errors in $I_{A A}$ is one of the main drawbacks in the study of possible jet modification in the small system collisions where the degree of suppression could be very small. The double ratio, $R_{I}$, of away to near side yield [3] substantially decreases the systematic errors. The $R_{I}$ is defined as,

$$
R_{I} \equiv \frac{Y_{a w a y}^{A A} / Y_{\text {near }}^{A A}}{Y_{a w a y}^{p p} / Y_{\text {near }}^{p p}},
$$

Taking the double ratio and choosing to integrate over whole hemispheres, results in a number of benefits which substantially reduce the systematic errors. The Fig. 3 shows a direct comparison
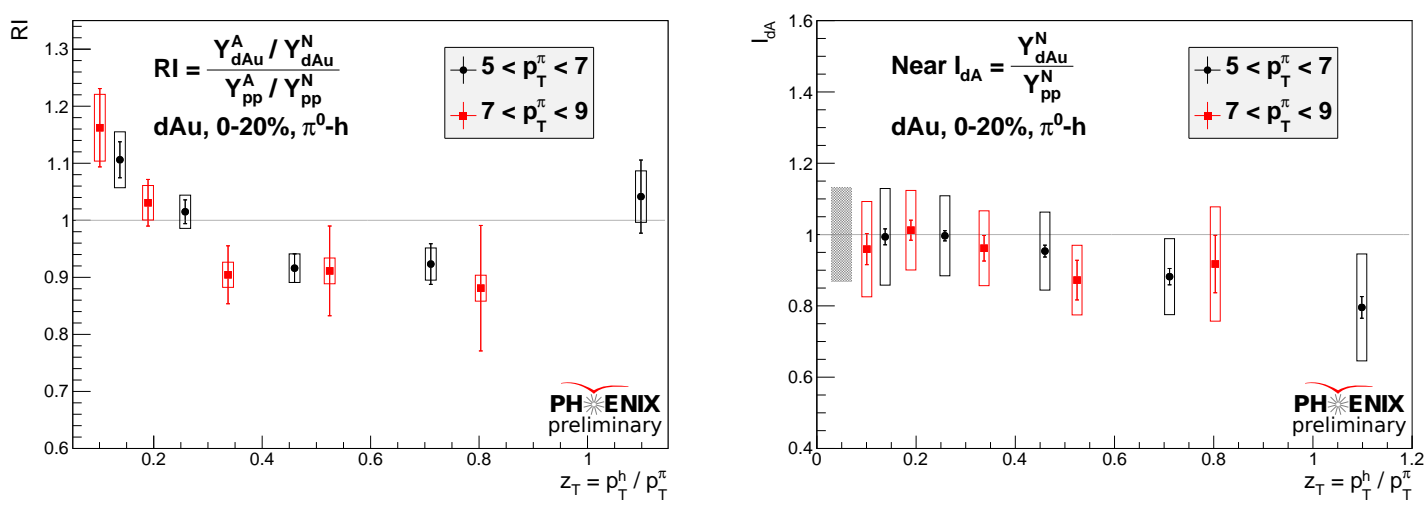

Figure 3: Left: $R_{I}$ vs. $z_{T}$ in $d+\mathrm{Au}$ collisions at $\sqrt{s_{N N}}=200 \mathrm{GeV}$. $z_{T}$ is ratio of transverse momentum of partner particle to that of trigger particle. Right: $I_{A A}$ vs. $z_{T}$ in $d+\mathrm{Au}$ collisions at $\sqrt{s_{N N}}=200 \mathrm{GeV}$. Both are at $0-20 \%$ centrality. These figures are taken from [3].

between $I_{A A}$ vs. $R_{I}$ in $d+\mathrm{Au}$ collisions. There is a clear reduction of uncertainties in $R_{I}$ compared to $I_{A A}$. Therefore, $R_{I}$ can be more sensitive to small levels of suppression or small cold nuclear effects. 
Furthermore, $R_{I}$ in $d+$ Au collisions shows small but statistically significant high $z_{T}$ suppression and low $z_{T}$ enhancement. This interesting behavior of $R_{I}$ in $d+\mathrm{Au}$ motivated us to confirm the result with another set of data. Since ${ }^{3} \mathrm{He}+\mathrm{Au}$ is the next bigger system after $d+\mathrm{Au}$, we analyzed the ${ }^{3} \mathrm{He}+\mathrm{Au}$ data at $200 \mathrm{GeV}$.
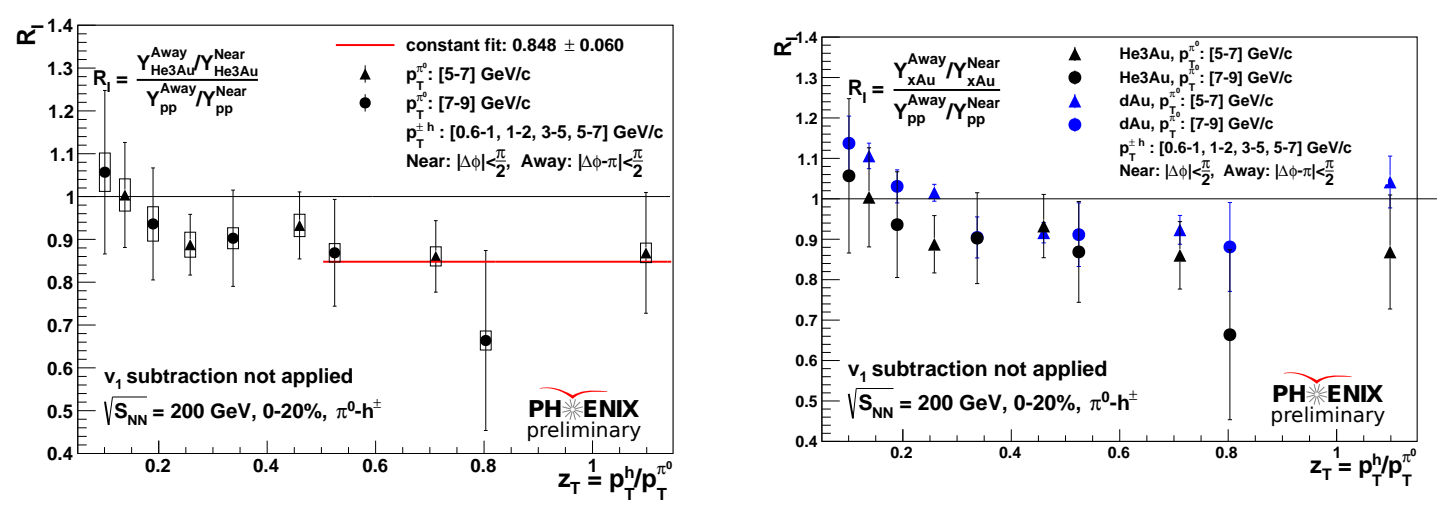

Figure 4: Left: $R_{I}$ vs. $z_{T}$ in $H e 3+A u$ collisions at $\sqrt{s_{N N}}=200 \mathrm{GeV}$. Right: Comparison of $R_{I}$ of He3+Au collisions to that of $d+\mathrm{Au}$. The blue points are of $d+\mathrm{Au}$ while black points are of ${ }^{3} \mathrm{He}+\mathrm{Au}$.

In Fig. 4, the $R_{I}$ of ${ }^{3} \mathrm{He}+\mathrm{Au}$ collisions confirms the behavior of $R_{I}$ of $d+\mathrm{Au}$ collisions. There is significant suppression at high $z_{T}$. The $R_{I}$ of ${ }^{3} \mathrm{He}+\mathrm{Au}$ collisions shows a rise at small $z_{T}$ similar to that of $d+\mathrm{Au}$ collisions although the uncertainties are too large to confirm the behavior with significance. Different tests viz; hydrodynamics $\left(v_{2}, v_{3}\right)$, enhanced nuclear $k_{T}$, initial state nPDFs etc. could not reproduce the modification in $R_{I}$ [3]. In order to find out how statistically significant is system size dependence of high $z_{T}$ suppression, we took the ratio of $R_{I}$ of ${ }^{3} \mathrm{He}+\mathrm{Au}$ collisions to that of $d+\mathrm{Au}$ collisions at high $z_{T}$. The ratio is as shown in Fig. 5.

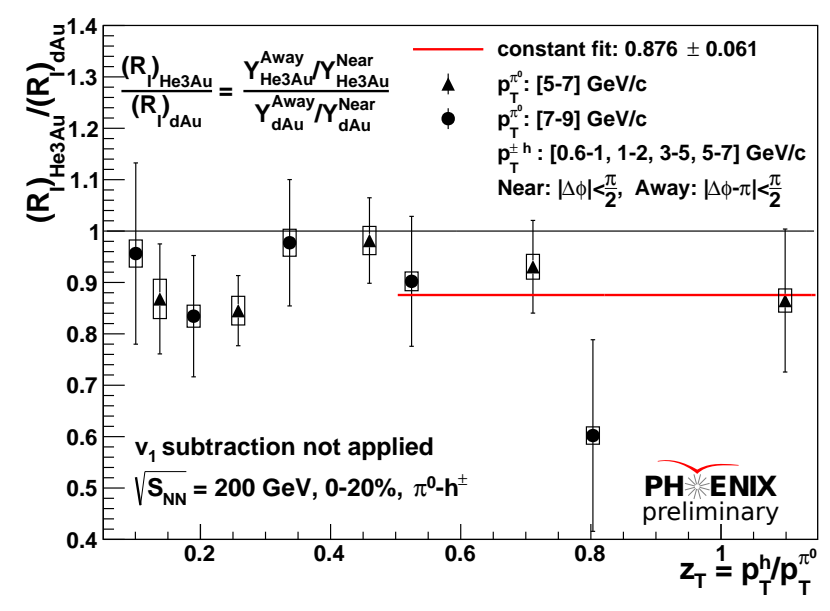

Figure 5: The ratio of $R_{I}$ in ${ }^{3} \mathrm{He}+\mathrm{Au}$ collisions to that in $d+\mathrm{Au}$ collisions.

The Fig. 5 shows that high $z_{T}$ suppression in ${ }^{3} \mathrm{He}+\mathrm{Au}$ collisions is about $12 \%$ larger than that in $d+\mathrm{Au}$ collisions with at least 2 sigma significance. 


\section{Conclusion}

The first measurement in isolated photon-hadron correlation in different centrality regions in $\mathrm{Au}+\mathrm{Au}$ collisions and $\pi^{0}$-hadron correlation in $d+\mathrm{Au} \&{ }^{3} \mathrm{He}+\mathrm{Au}$ collisions are presented. Isolation cut allows more precise analysis of semi-peripheral and peripheral centralities in Au+Au collisions. The low $z_{T}$ relative enhancement in $I_{A A}$ from isolated photon-hadron correlation is monotonically increasing with centrality.

The $R_{I}$ from $\pi^{0}$-hadron correlation in ${ }^{3} \mathrm{He}+\mathrm{Au}$ collisions shows similar suppression at high $z_{T}$ and similar shape overall to that in $d+\mathrm{Au}$ collisions. The ordering of increasing suppression with volume size is confirmed with $R_{I}$ of ${ }^{3} \mathrm{He}+\mathrm{Au}$ collisions being more suppressed than that of $d+\mathrm{Au}$ collisions.

\section{References}

[1] PHENIX Collaboration Phys.Rev. D82 072001 (2010).

[2] T. Danley, Photon-Related Elliptic Azimuthal Asymmetry and Photon-Hadron Correlations with an Isolation Cut in $\mathrm{Au}+\mathrm{Au}$ Collisions at $\sqrt{s_{N N}}=200 \mathrm{GeV}$ at RHIC-PHENIX, Ph.D. thesis, Ohio University (2018).

[3] B. Xia, $\pi^{0}-h$ Jet Correlation in $d+$ Au Collisions at $\sqrt{s_{N N}}=200 \mathrm{GeV}, \mathrm{Ph}$.D. thesis, Ohio University (2014).

[4] A. Sickles, M. P. McCumber, and A. Adare, Phys. Rev. C81, 014908 (2010). 